\title{
Frequency Packing for Interference Alignment-based Cognitive Dual Satellite Systems
}

\author{
Symeon Chatzinotas, Shree Krishna Sharma and Björn Ottersten \\ SnT - securityandtrust.lu, University of Luxembourg \\ Email:\{symeon.chatzinotas, shree.sharma, bjorn.ottersten\}@uni.lu
}

\begin{abstract}
Interference Alignment (IA) has been considered a promising technique for spectral coexistence of different wireless systems in an underlay cognitive mode. Furthermore, Frequency Packing (FP) can be considered as an important technique for enhancing the spectrum efficiency in spectrum-limited satellite applications. In this paper, we consider a spectral coexistence scenario of a multibeam satellite and a monobeam satellite with the monobeam satellite as primary and the multibeam satellite as secondary. In this context, this paper focuses on examining the effect of FP on the performance of multi-carrier based IA technique. For this purpose, different IA techniques such as coordinated IA, uncoordinated IA and static IA have been considered. The effect of FP on the performance of different IA techniques in the considered scenario is evaluated in terms of system sum rate and primary rate protection ratio. It is shown that the system sum rate increases with the FP factor for all the techniques and the primary rate is perfectly protected with the coordinated IA technique even with dense FP.
\end{abstract}

Index Terms: Interference Alignment, Dual Satellite System, Frequency Packing, Spectral Coexistence

\section{INTRODUCTION}

Next generation satellite communications systems target enhanced throughput and higher spectral efficiency. To enhance system capacity, satellite systems have moved from payloads generating a single beam to multi-beam platforms [1]. Multiple beams can be employed instead of a single global beam in order to enhance the capacity through spatial frequency reuse [2]. However, most satellite systems use monobeam systems and the deployment of new multibeam systems needs additional bandwidth which is extremely scarce. In this context, multibeam satellites have to coexist with the traditional monobeam satellites in order to utilize the existing spectrum more efficiently. This need has led to the concept of cognitive Satellite Communications (SatComs) which allows the spectral coexistence of two systems, primary and secondary. The spectrum coexistence literature is more mature in the terrestrial context but has received limited attention in the satellite context. Recent contributions exploiting spectrum sharing opportunities in the context of cognitive SatComs include [3-9]. Furthermore, most of the current contributions in this context focus on hybrid coexistence scenario of satellite and terrestrial systems and only a few contributions address dual satellite coexistence scenarios [4,9-12].
The spectral coexistence of two satellite systems can be modeled as a Cognitive Radio (CR) network with interference channels between primary and secondary systems. The operation of the primary network usually follows a predefined standard and should not be degraded, while the secondary network should employ advanced communication techniques to exploit the underutilized dimensions in the signal space. Depending on the level of interference between primary and secondary systems, different cognitive techniques can be employed [3]. The most common cognitive techniques in the literature can be categorized into interweave or Spectrum Sensing (SS), underlay, overlay and database techniques. In SS only techniques, Secondary Users (SUs) are allowed to transmit whenever Primary Users (PUs) are not active in that specific band, whereas in underlay techniques, SUs are allowed to transmit as long as they respect the interference constraint of the PUs. When the strength of secondary interference to the primary is comparable to the desired signal, treating as noise is not an option because of interference constraints involved while decoding and the requirement of complex primary receivers. In this context, Interference Alignment (IA) has received an important attention as an interference mitigation tool in the CR research community $[11,13,14]$. The concept behind IA is that signals can be designed in such a way that they cast overlapping shadows at the non-intended receivers and remain distinguishable at the desired receivers.

In this direction, IA technique has been shown to achieve the degrees of freedom for a range of interference channels $[15,16]$. The fundamental assumptions which make the IA technique feasible are that there are multiple available dimensions (space, frequency, time or code) and that the transmitter is aware of the Channel State Information (CSI) towards the non-intended receiver. It should be noted that IA can be classified as an underlay cognitive technique [17] since it deals with interference mitigation towards the primary system in frequency coexistence scenarios. In [18], different IA techniques such as coordinated, uncoordinated and static IA techniques have been applied for the spectral coexistence scenario of small cells and a macro cell considering spatial (antenna) dimension for the alignment purpose. Furthermore, the IA technique has been investigated in multi-carrier systems in various settings [11,19-22]. In [11], the performance of 
different IA techniques is evaluated for multi-carrier cognitive dual satellite systems in order to mitigate the interference of multibeam satellite terminals towards a monobeam satellite.

Spectral efficiency can be further enhanced by increasing the packing density of the sub-carriers. The term Frequency Packing (FP) refers to reducing spacing between adjacent signals in the frequency domain, while employing advanced techniques for suppressing or exploiting the additionally induced interference. In [23], it has been concluded that the spectral efficiency of linear modulations with finite order constellations can be improved by reducing the spacing between adjacent signals, both in time and frequency domains. This technique has appeared in the literature in several names such as Fast OFDM (FOFDM) [24, 25], Spectrally efficient FDM (SEFDM) [26], M-ary amplitude shift keying (M-ary ASK), High Compactions Multi-carrier Communications (HC-MCM) [27] etc. In [25], the first experimental demonstration of optical Fast-OFDM (FOFDM) system has been carried out with a reduced sub-carrier spacing equal to the symbol rate. In [24], a novel DFT-based implementation for optical FOFDM has been carried out and in [28], a novel algorithm for allocating and packing time-frequency slots in a jitter-aware fashion has been proposed using the groups of evenly spaced slots.

In this paper, we extend the contributions of [18] and [11] by examining the effect of FP on different IA techniques. The multi-carrier IA technique presented in [11] takes into account of the Adjacent Carrier Interference (ACI) but does not evaluate the performance of it on different IA techniques. The ACI may result from the FP as well as from imperfect bandpass filters. In this paper, we are interested in evaluating the effect of FP on the performance of different IA techniques assuming perfect bandpass filters. The performance of different IA techniques with the FP is evaluated in the considered scenario in terms of the sum rate and the primary rate protection ratio.

This paper is organized as follows: Section II describes the considered system and signal models. Section III describes different coexistence techniques including three different types of IA techniques. Section IV presents the proposed FP technique for the considered multi-carrier system. Section $V$ presents the considered performance metrics and evaluates the effect of FP on the system performance. Section VI concludes the paper.

\section{A. Notation}

Throughout this article, $\mathbb{E}[\cdot]$ denotes expectation, $(\cdot)^{T}$ and $(\cdot)^{\dagger}$ denote the transpose and the conjugate transpose respectively, $\odot$ denotes the Hadamard product, $\otimes$ denotes the Kronecker product, $\mathbf{I}_{n}$ denotes a $n \times n$ identity matrix and $\mathbb{I}_{n \times m}$ denotes a $n \times m$ matrix of ones.

\section{System AND Signal Model}

\section{A. System Model}

Let us consider a monobeam satellite (SAT1) and a multibeam satellite (SAT2) serving the same coverage area as shown in Fig. 1. These satellites are assumed to be connected to different gateways. We consider the monobeam system as primary and the multibeam system as secondary with both satellites operating in normal return mode. The monobeam and multibeam satellites can be adjacent or even colocated in terms of orbital slots. The cognition between these two systems can be achieved with the help of a high speed signalling link (i.e., microwave or optical fiber) between their gateways. Furthermore, we consider a single monobeam satellite terminal (ST1), $N$ number of multibeam satellite terminals (ST2s) served by $N$ beams of the SAT2. Multibeam joint processing is considered at the gateway of SAT2 to decode the received signals from ST2s jointly [2]. Since a single gateway is responsible for processing the transmitted and received signals corresponding to a large geographic area, the application of joint processing techniques in satellite context is centralized. After scheduling, we consider that one ST1 and $N$ number of ST2s are transmitting simultaneously in a single slot over a common spectrum band. In this context, the IA technique can be applied at the mutlibeam satellite terminals to mitigate the interference towards the primary satellite as illustrated in [11].

Furthermore, we consider that all the satellite terminals use multi-carrier transmission scheme and the IA is employed at the ST2s over $L=M+1$ carriers. We consider that $M$ number of symbols are transmitted by the ST1 and 1 symbol per ST2 is transmitted by spreading across all the carriers. Furthermore, it should be noted that the ST1 sends $M$ symbols over $M$ subcarriers whereas each ST2 sends 1 symbol over $L$ subcarriers. To suppress the interference caused by the ST2s using IA technique, the CSI towards the SAT1 is required and we assume that this CSI can be acquired at the ST2s by listening to the pilot signals broadcasted from the gateway of the primary system. In this context, we assume time division duplex mode of operation and for a satellite system with frequency division duplex mode of operation, an alternative way of acquiring CSI should be investigated since uplink CSI can not be derived from the downlink pilots in FDD mode.

\section{B. Signal and Channel Model}

In the proposed system model, we consider that frequency packing is employed at the transmitter to enhance the spectral efficiency. Due to this phenomenon, adjacent carrier interference is introduced which is described in detail in Section IV. We assume perfect bandpass receive filters for simplicity of analysis ${ }^{1}$.

The received signal at the primary receiver i.e., SAT1 can be written as:

$$
\mathbf{y}_{p}=\mathbf{H} \mathbf{x}+\sum_{i=1}^{N} \mathbf{F}_{i} \mathbf{x}_{i}+\mathbf{z}_{p},
$$

where $\mathbf{y}_{p}$ is the $L \times 1$ received symbol vector, $\mathbf{x}$ and $\mathbf{x}_{i}$ are the $M \times 1$ and $L \times 1$ transmitted symbol vectors from the ST1 and the $i$ th ST2 respectively, and $\mathbf{z}_{p}$ is the receiver noise. The input signals $\mathbf{x}, \mathbf{x}_{i}$ are assumed to be Gaussian and obey the following sum power constraints: $\mathbb{E}\left[\mathbf{x}^{\dagger} \mathbf{x}\right] \leq \gamma_{p} M$

\footnotetext{
${ }^{1}$ We need to consider the combined effect of the ACI introduced due to packing and due to band pass filters in case of imperfect bandpass filters.
} 


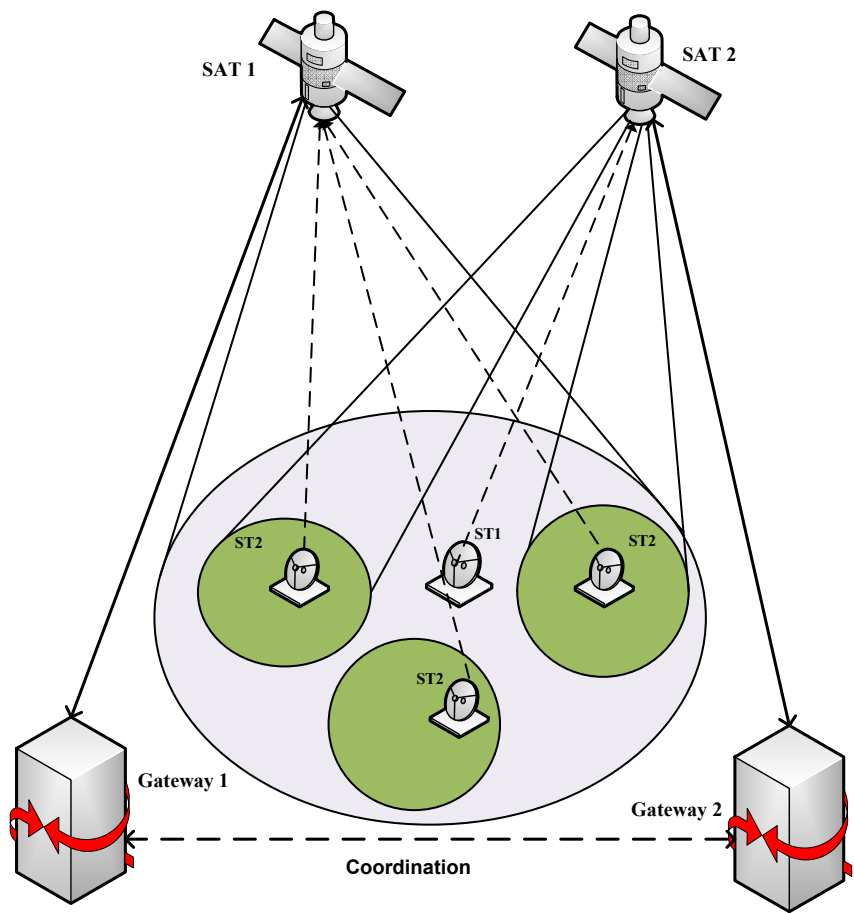

Fig. 1: Graphical representation of the considered dual satellite coexistence system. The dotted lines represent the interfering links..

and $\mathbb{E}\left[\mathbf{x}_{i}^{\dagger} \mathbf{x}_{i}\right] \leq \gamma_{s} L, \gamma_{p}$ and $\gamma_{s}$ being the transmit SNR of the primary and secondary systems respectively. The $L \times M$ matrix $\mathbf{H}$ represents the channel gains between the SAT1 and the ST1 while the $L \times L$ matrix $\mathbf{F}_{i}$ represents the channel gains between the SAT1 and $i$ th ST2. To simplify notations, all $\mathbf{F}_{i}$ are grouped into a single $L \times N L$ matrix $\mathbf{F}=\left[\mathbf{F}_{1} \ldots \mathbf{F}_{N}\right]$. The received signal at the joint processor of the SAT2 gateway can be written as:

$$
\mathbf{y}_{s}=\sum_{i=1}^{N} \tilde{\mathbf{F}}_{i} \mathbf{x}_{i}+\tilde{\mathbf{H}} \mathbf{x}+\mathbf{z}_{s},
$$

where $\mathbf{y}_{s}$ is the $N L \times 1$ received symbol vector and $\mathbf{z}_{s}$ is the receiver noise. The $N L \times M$ channel matrix $\tilde{\mathbf{H}}$ represents the channel gains between all SAT2 beams and the ST1 while the $N L \times L$ channel matrix $\tilde{\mathbf{F}}_{i}$ represents the channel gains between all SAT2 beams and the $i$ th ST2. We group all $\tilde{\mathbf{F}}_{i}$ into a single $N L \times N L$ matrix $\tilde{\mathbf{F}}=\left[\tilde{\mathbf{F}}_{1} \ldots \tilde{\mathbf{F}}_{N}\right]$.

We assume that each carrier goes through independent flatfading channels. The multi-carrier channel matrix with ACI for the $i$ th satellite link for $L$ number of carriers can be written as:

$$
\mathbf{H}=\left[\begin{array}{cccc}
h_{1} & \mu h_{2} & \ldots & 0 \\
\mu h_{1} & h_{2} & \ldots & 0 \\
0 & \mu h_{2} & \ldots & 0 \\
\vdots & \vdots & \vdots & \vdots \\
0 & 0 & h_{L-1} & \mu h_{L} \\
0 & 0 & \mu h_{L-1} & h_{L}
\end{array}\right]
$$

where $\mu$ represents correlation amplitude between two subcarriers and it characterizes the effect of intercarrier interference as illustrated in Section IV. The parameter $h_{i}$ represents the Rician fading channel coefficient and is given by;

$$
h_{i}=\left(\sqrt{\frac{K}{K+1}} l+\sqrt{\frac{1}{K+1}} g_{i}\right)
$$

where $K$ is the Rician factor, $l$ is a deterministic parameter representing the line of sight component and $g_{i}$ is a c.c.s. i.i.d. element for the $i$ th satellite link representing the Rayleigh fading coefficient. The channel matrix between the SAT1 and the $i$ th ST2 can be written as:

$$
\mathbf{F}_{i}=\alpha_{i} \mathbf{D}_{i}
$$

where $\alpha_{i}$ is the beam gain coefficient between the SAT1 and the $i$ th SAT2 and $\mathbf{D}_{i}$ has similar structure as $\mathbf{H}$. As a result,

$$
\mathbf{F}=\left(\boldsymbol{\alpha}^{T} \otimes \mathbb{I}_{L \times L}\right) \odot \mathbf{D}
$$

with $\boldsymbol{\alpha}=\left[\alpha_{1} \ldots \alpha_{N}\right]^{T}$ and $\mathbf{D}=\left[\mathbf{D}_{1} \ldots \mathbf{D}_{N}\right]$. It is assumed that the fading coefficients are independent across block matrices $\mathbf{D}_{i}$. In addition, the channel matrix between SAT2 and ST1 can be written as:

$$
\tilde{\mathbf{H}}=\left(\boldsymbol{\beta} \otimes \mathbb{I}_{L \times M}\right) \odot \mathbf{P},
$$

where $\boldsymbol{\beta}=\left[\beta_{1} \ldots \beta_{N}\right]^{T}$ includes beam gain coefficients between SAT2 and ST1 and $\mathbf{P}=\left[\mathbf{P}_{1} \ldots \mathbf{P}_{N}\right]^{T}$ is a block matrix with each $\mathbf{P}_{i}$ having similar structure as $\mathbf{H}$. Since we consider transmission using $M$ out of $L$ carriers, excluding the last column from $\mathbf{H}$ provides similar structure as $\mathbf{P}_{i}$. Similarly, the channel matrix between SAT2 and the $i$ th ST2 can be written as:

$$
\tilde{\mathbf{F}}_{i}=\left(\boldsymbol{\beta}_{i} \otimes \mathbb{I}_{L \times L}\right) \odot \mathbf{S}_{i},
$$

where $\boldsymbol{\beta}_{i}$ contains the beam gain coefficient between SAT2 and the $i$ th ST2 and $\mathbf{S}_{i}$ has similar structure as $\mathbf{H}$. As a result,

$$
\tilde{\mathbf{F}}=\left(\mathbf{B} \otimes \mathbb{I}_{L \times L}\right) \odot \mathbf{S},
$$

with $\mathbf{B}=\left[\boldsymbol{\beta}_{1} \ldots \boldsymbol{\beta}_{N}\right]$ and the block matrix $\mathbf{S}$ given by;

$$
\mathbf{S}=\left[\begin{array}{cccc}
\mathbf{S}_{11} & \mathbf{S}_{12} & \cdots & \mathbf{S}_{1 N} \\
\mathbf{S}_{21} & \mathbf{S}_{22} & \cdots & \mathbf{S}_{2 N} \\
& & \ddots & \\
\mathbf{S}_{N 1} & \mathbf{S}_{N 1} & \cdots & \mathbf{S}_{N N}
\end{array}\right]
$$

where each block $\mathbf{S}_{i j}$ follows the similar structure as $\mathbf{H}$ and it indicates the fading coefficients of the multi-carrier channel between the $i$ th ST2 and the $j$ th beam of SAT2.

The beam gain of the satellite link in all the above cases are evaluated based on following expression [3]:

$$
b(m, k)=g_{\max } \cdot\left(\frac{J_{1}(u(m, k))}{2 u(m, k)}+36 \frac{J_{3}(u(m, k))^{2}}{u(m, k)^{3}}\right)^{2},
$$

where $b(m, k)$ represents the beam gain of the $k$ th beam for the $m$ th terminal position, $u(m, k)=2.01723 \sin (\theta(m, k)) / \theta_{3 \mathrm{~dB}}$, $J_{i}$ is the first kind of Bessel's function of order $i$, and $g_{\max }$ is the maximum antenna gain. $\theta_{3 \mathrm{~dB}}$ is the $3 \mathrm{~dB}$ angle and $\theta(m, k)$ represents the angular separation of the $m$ th terminal 
from the $k$ th beam center position with respect to the satellite.

\section{Coexistence Techniques}

In this section, firstly, we provide the formulations of the capacity expressions which we are going to use for evaluating the performance of different techniques in the considered scenario. In the presence of cochannel interference, the inputoutput relation for a Multiple Input Multiple Output (MIMO) system can be written as:

$$
\mathbf{y}=\mathbf{H} \mathbf{x}+\mathbf{H}_{c} \mathbf{x}_{c}+\mathbf{z}
$$

with $\mathbb{E}\left[\mathbf{x} \mathbf{x}^{\dagger}\right]=\gamma_{p} \mathbf{I}$ and $\mathbb{E}\left[\mathbf{x}_{c} \mathbf{x}_{c}^{\dagger}\right]=\gamma_{s} \mathbf{I}$, where $\mathbf{x}_{c}$ is a Gaussian vector transmitted by an interfering cochannel terminal. Then the capacity of a MIMO channel with inputoutput relation given by (12) can be written as [29]:

$$
C=\log \operatorname{det}\left(\mathbf{I}+\gamma_{p} \mathbf{H} \mathbf{H}^{\dagger} \mathbf{R}^{-1}\right),
$$

where the term $\mathbf{R}^{-1}$ includes the effect of cochannel interference and $\mathbf{R}$ can be written as:

$$
\mathbf{R}=\mathbb{E}\left[\tilde{\mathbf{z}} \tilde{\mathbf{z}}^{\dagger}\right]=\mathbf{I}+\gamma_{s} \mathbf{H}_{c} \mathbf{H}_{c}^{\dagger}
$$

with $\tilde{\mathbf{z}}=\mathbf{H}_{c} \mathbf{x}_{c}+\mathbf{z}$. In the absence of cochannel interference, the expression (13) simply reduces to the following form.

$$
C=\log \operatorname{det}\left(\mathbf{I}+\gamma_{p} \mathbf{H} \mathbf{H}^{\dagger}\right)
$$

In the following paragraphs, we review different possible coexistence techniques for the considered scenario including IA-based techniques.

\section{A. No Mitigation}

In this case, we assume that no interference mitigation techniques have been introduced and uniform power allocation is applied across the carriers of the satellite terminals. This is an interference-limited coexistence scenario. The primary throughput in presence of the secondary, denoted by $\mathrm{C}_{\mathrm{ps}}$, can be written as:

$$
\mathrm{C}_{\mathrm{ps}}=\mathbb{E}\left[\log \operatorname{det}\left(\mathbf{I}_{L}+\frac{\gamma_{p}}{M} \mathbf{H} \mathbf{H}^{\dagger}\left(\mathbf{I}_{L}+\frac{\gamma_{s}}{L} \mathbf{F} \mathbf{F}^{\dagger}\right)^{-1}\right)\right],
$$

Similarly, the secondary throughput in the presence of the primary, denoted by $\mathrm{C}_{\mathrm{sp}}$, can be written as:

$$
\mathrm{C}_{\mathrm{sp}}=\mathbb{E}\left[\log \operatorname{det}\left(\mathbf{I}_{N L}+\frac{\gamma_{s}}{L} \tilde{\mathbf{F}} \tilde{\mathbf{F}}^{\dagger}\left(\mathbf{I}_{N L}+\frac{\gamma_{p}}{M} \tilde{\mathbf{H}} \tilde{\mathbf{H}}^{\dagger}\right)^{-1}\right)\right] .
$$

In both the above cases, the second term represents the cochannel interference.

\section{B. Resource Division}

In this technique, we assume that the available resource is split into two in order to allow the interference free parallel operation of primary and secondary systems. The orthogonalization is considered in the time domain for the considered multi-carrier scenario. In this case, the primary throughput, denoted by $\hat{\mathrm{C}}_{p r}$, can be written as:

$$
\hat{\mathrm{C}}_{\mathrm{pr}}=\frac{1}{2} \mathbb{E}\left[\log \operatorname{det}\left(\mathbf{I}_{L}+\frac{2 \gamma_{p}}{M} \mathbf{H} \mathbf{H}^{\dagger}\right)\right],
$$

while the secondary throughput, denoted by $\hat{\mathrm{C}}_{s r}$, can be written as:

$$
\hat{\mathrm{C}}_{\mathrm{sr}}=\frac{1}{2} \mathbb{E}\left[\log \operatorname{det}\left(\mathbf{I}_{N L}+\frac{2 \gamma_{s}}{L} \tilde{\mathbf{F}} \tilde{\mathbf{F}}^{\dagger}\right)\right]
$$

\section{Interference Alignment}

In this technique, IA is employed at all the ST2s towards the SAT1 and interference is filtered out at the SAT1 by using IA vector. In this case, the primary throughput, denoted by $\overline{\mathrm{C}}_{\mathrm{ps}}$, can be written as:

$$
\overline{\mathrm{C}}_{\mathrm{ps}}=\mathbb{E}\left[\log \operatorname{det}\left(\mathbf{I}_{M}+\frac{\gamma_{p}}{M} \overline{\mathbf{H}} \overline{\mathbf{H}}^{\dagger}\right)\right]
$$

where $\overline{\mathbf{H}}$ is the equivalent channel matrix after IA filtering. For the SAT2, the interference coming from the ST1 has to be tolerated and thus secondary throughput, $\overline{\mathrm{C}}_{\mathrm{sp}}$, can be written as:

$$
\overline{\mathrm{C}}_{\mathrm{sp}}=\mathbb{E}\left[\log \operatorname{det}\left(\mathbf{I}_{N L}+\frac{\gamma_{s}}{L} \overline{\mathbf{F}} \overline{\mathbf{F}}^{\dagger}\left(\mathbf{I}_{N L}+\frac{\gamma_{p}}{M} \tilde{\mathbf{H}} \tilde{\mathbf{H}}^{\dagger}\right)^{-1}\right)\right],
$$

where $\overline{\mathbf{F}}$ is the equivalent channel matrix including precoding.

1) IA and Filtering: Let us assume a $L \times 1$ non-zero reference vector $\mathbf{v}$ along which the interference should be aligned. It should be noted that ST2s are assumed to know the alignment direction $\mathbf{v}$ and to have the perfect CSI knowledge towards the SAT1. The alignment direction for each group of terminals can be predetermined or alternatively coordinated with the help of signaling from the intended gateway. In this context, the following precoding scheme is employed to align the interference.

$$
\mathbf{x}_{i}=\mathbf{w}_{i} x_{i}=\left(\mathbf{F}_{i}\right)^{-1} \mathbf{v} v_{i} x_{i}
$$

where $\|\mathbf{v}\|^{2}=L$ and the scaling variable $v_{i}$ is introduced to ensure that the input power constraint is not violated for each ST2. The cochannel interference then can be expressed as:

$$
\sum_{i=1}^{N} \mathbf{F}_{i} \mathbf{x}_{i}=\sum_{i=1}^{N} \mathbf{F}_{i}\left(\mathbf{F}_{i}\right)^{-1} \mathbf{v} v_{i} x_{i}=\mathbf{v} \sum_{i=1}^{N} v_{i} x_{i} .
$$

It can be easily observed that the interference has been aligned across $\mathbf{v}$ and it can be removed using a $M \times L$ zero-forcing filter $\mathbf{Q}$ designed so that $\mathbf{Q}$ is a truncated unitary matrix [15] and $\mathbf{Q v}=\mathbf{0}$. After filtering, the $M \times 1$ received signal vector at the SAT1, denoted by $\overline{\mathbf{y}}_{1}$, can be expressed as:

$$
\overline{\mathbf{y}}_{1}=\overline{\mathbf{H}} \mathbf{x}+\overline{\mathbf{z}}_{1},
$$

where $\overline{\mathbf{H}}=\mathbf{Q H}$ is the $M \times M$ filtered channel matrix. Assuming that the system operates in the high SNR region and is therefore interference limited, the effect of the Additive White Gaussian Noise (AWGN) colouring $\overline{\mathbf{z}}_{1}=\mathbf{Q} \mathbf{z}_{1}$ can be ignored, namely $\mathbb{E}\left[\overline{\mathbf{z}}_{1} \overline{\mathbf{z}}_{1}^{H}\right]=\mathbf{I}$. Furthermore, the received signal at the joint processor of the SAT2 gateway is:

$$
\overline{\mathbf{y}}_{2}=\sum_{i=1}^{N} \overline{\mathbf{F}}_{i} x_{i}+\tilde{\mathbf{H}} \mathbf{x}+\mathbf{z}_{2},
$$


where $\overline{\mathbf{F}}_{i}=\tilde{\mathbf{F}}_{i}\left(\mathbf{F}_{i}\right)^{-1} \mathbf{v} v_{i}$ are the equivalent $N L \times 1$ channel matrices including precoding. In the following paragraphs, we describe three different IA approaches. The detailed mathematical formulations of these techniques and the theoretical proof that the coordinated approach can perfectly protect the primary rate can be found in [11].

2) IA Types: a) Static IA: In this approach, $\mathbf{v}$ is predefined and does not depend on the channel state. It can be noted that this is quite static but also a simple solution which assumes no coordination in the network. The disadvantage is that a large amount of received power may be filtered out since the IA direction may be aligned with one of the strong eigenvectors of the random SAT1-ST1 channel.

b) Coordinated IA: In this approach, the primary and secondary systems coordinate to exchange the CSI information and the alignment vector. The selection of $\mathbf{v}$ takes place at the SAT1 and is subsequently communicated to the ST2s. It is assumed that the channel coherence time is adequate for the alignment direction to be fed back and used by the ST2s.

c) Uncoordinated IA: This approach assumes that the primary and the secondary systems do not coordinate. Furthermore, ST2s are aware of their CSI towards the SAT1 but have no information about the CSI of the ST1. In this context, the ST2s select $\mathbf{v}$ in order to maximize the secondary throughput. Subsequently, the SAT1 senses the $\mathbf{v}$ and applies the appropriate filter $\mathbf{Q}$.

\section{Proposed FP Technique}

In this section, we provide the theoretical analysis of the FP technique proposed for the considered scenario. For FP purpose, non-orthogonal carriers need to be generated at the transmitter. The FDM system using non-orthogonal carriers can be considered as an SEFDM system. The SEFDM system can provide significant spectral savings but it requires complex detection process due to Intercarrier Interference (ICI) caused by the loss of orthogonality between subcarriers. Therefore, it is extremely important to study the effect of ICI on the system performance and to characterize it using related parameters.

The SEFDM symbol $x(t)$ is composed by multiplexing $N$ input complex symbols, let us denote by $s$, onto the parallel subcarriers and can be written as [26]:

$$
x(t)=\frac{1}{\sqrt{T}} \sum_{n=0}^{N-1} s_{n} \exp (j 2 \pi n \delta t / T),
$$

where $s_{n}$ is the symbol modulated on the $n$th subcarrier, $T$ is the SEFDM symbol duration, $\delta$ denotes the bandwidth compression factor. The parameter $\delta$ can be defined as:

$$
\delta=\Delta f T
$$

where $\Delta f$ is the frequency separation between adjacent subcarriers. Let $N_{c}$ denote the number of subcarriers at the value of $\delta=1$ i.e., the total number of orthogonal carriers that can be placed at a given bandwidth without FP. After applying FP, the modified number of carriers which can be fit into the same available bandwidth becomes $N_{c p}=N_{c} / \delta$. We define the FP factor $\rho$ as $\rho=1-\delta$ for simplicity of analysis.
The ACI considered for the considered multi-carrier system in Section II can be modeled as a function of bandwidth compression factor and the number of subcarriers. The ACI caused by the loss of orthogonality can be calculated by finding the correlation between the subcarriers. Let us consider two arbitrary SEFDM subcarriers denoted by $\psi_{m}(t)$ and $\psi_{n}(t)$, which can be defined as:

$$
\psi_{i}(t)=e^{\frac{j 2 \pi \delta i t}{T}}
$$

where $t \in(0, T)$ and $i=0,1, \cdots, N-1$. The correlation between these subcarriers can be defined as:

$$
\mu(m, n)=\left\langle\psi_{m}(t), \psi_{n}(t)\right\rangle,
$$

where $\langle\cdot\rangle$ denotes the inner product. The value of $\mu(m, n)$ can be further calculated as [26]:

$$
\begin{aligned}
\mu(m, n) & =\frac{1}{T} \int_{0}^{T} \psi_{m}(t) \psi_{n}^{*}(t) \\
& =\operatorname{sinc}(2 \delta(m-n))+j \sin (\pi \delta(m-n)) \operatorname{sinc}(\delta(m-n))
\end{aligned}
$$

The amplitude of $\mu(m, n)$ from the above equation can be written as:

$$
|\mu(m, n)|=|\operatorname{sinc}(\delta(m-n))|,
$$

where $|\cdot|$ denotes the absolute operation. In this paper, we consider the effect of ICI between adjacent carriers only i.e., $\mathrm{ACI}$ and in this case, the factor $m-n$ reduces to 1 . Therefore, the correlation amplitude representing the effect of ACI caused by the FP phenomenon i.e., $\mu$ can be written as:

$$
\mu=|\operatorname{sinc}(\delta)| \text {. }
$$

\section{NUMERICAL RESUltS}

In this section, we present numerical results in order to show the effect of FP on the performance of the considered IA techniques for the considered coexistence scenario.

\section{A. Performance Metrics}

In order to evaluate the system performance, three different metrics are considered. The system sum-rate capacity is denoted by $\mathrm{C}_{\mathrm{sys}}$ and can be defined as:

$$
\mathrm{C}_{\mathrm{sys}}=\mathrm{C}_{\mathrm{ps}}+\frac{\mathrm{C}_{\mathrm{sp}}}{N}
$$

where $\mathrm{C}_{\mathrm{ps}}$ denotes the capacity of the monobeam satellite system in the presence of the multibeam satellite system and $\mathrm{C}_{\mathrm{sp}}$ denotes the capacity of the multibeam satellite system in the presence of the monobeam system. The secondary capacity is normalized by $N$ to take into account of per beam secondary capacity in the total system capacity expression. Subsequently, the primary rate protection ratio is denoted by $\mathrm{PR}$ and can be defined as:

$$
\mathrm{PR}=\frac{\mathrm{C}_{\mathrm{ps}}}{\mathrm{C}_{\mathrm{po}}},
$$

where $\mathrm{C}_{\mathrm{po}}$ denotes the primary only capacity and is given by;

$$
\mathrm{C}_{\text {po }}=\mathbb{E}\left[\log \operatorname{det}\left(\mathbf{I}_{L}+\frac{\gamma_{p}}{M} \mathbf{H H}^{\dagger}\right)\right] \text {. }
$$


TABLE I: Simulation Parameters

\begin{tabular}{l|c|c|} 
Parameter & Symbol & Value/Range \\
\hline Bandwidth compression factor & $\delta$ & $1-0.5$ \\
No. of ST2s/SAT2 beams & $N$ & 5 \\
Carriers used by ST1 without FP & $M$ & 9 \\
Carriers used by ST2 without FP & $L$ & 10 \\
Monobeam Radius & $R_{p}$ & $520 \mathrm{Km}$ \\
Multibeam Radius & $R_{s}$ & $165 \mathrm{Km}$ \\
ST1 Transmit Power & $P_{p}$ & $10 \mathrm{dBW}$ \\
ST2 Transmit Power & $P_{s}$ & $3.98 \mathrm{dBW}$ \\
Receiver Noise Power @ 5 MHz & $N_{0}$ & $-137 \mathrm{dBW}$ \\
Monobeam 3-dB Beamwidth & $B W_{p}$ & $0.82^{\circ}$ \\
Multibeam 3-dB Beamwidth & $B W_{s}$ & $0.26^{\circ}$ \\
Rician factor & $K$ & $12 \mathrm{~dB}$ \\
Free Space Path loss & $F L$ & $190 \mathrm{~dB}$ \\
Max Satellite Antenna Gain & $G_{\max }$ & $48 \mathrm{dBi}$ \\
Terminal Antenna Gain & $G_{T}$ & $5 \mathrm{~dB}$
\end{tabular}

\section{B. Simulation Environment}

While simulating the dual satellite coexistence scenario shown in Fig. 1, ST1 and ST2s are assumed to be uniformly distributed within the coverage area of the beams of SAT1 and SAT2, respectively. The beams of the multibeam satellite are assumed to be uniformly distributed within the coverage area of the monobeam satellite, emulating a beam hopping pattern or (electronically or mechanically) steerable beams. The Rician fading channel has been considered to reflect the practical satellite channel for L or S band mobile applications and multi-carrier transmission scheme has been considered for all the satellite terminals. The considered multi-carrier channel model considers the ACI effect and each non-zero component of the channel matrix has line of sight component and the fading component, representing the Rician fading coefficient. The simulation parameters used for producing the numerical results are presented in Table I. In the simulation setting, the bandwidth compression factor $\delta$ was varied from 1 to 0.5 and the corresponding FP factor and number of subcarriers were calculated using the expressions presented in Section IV. The value of $\mu$ was calculated using (32). We consider the following different techniques for comparing their performance while applying FP in the considered multicarrier transmission scheme: (i) Primary only (eqn. (35)), (ii) Resource division (eqn. (18) and (19)), (iii) IA static (Section III C), (iv) IA uncoordinated (Section III C), (v) IA coordinated (Section III C), and (vi) No mitigation (eqn. (16) and (17)). In the considered static approach, a random alignment vector was generated at the beginning and it was kept fixed for all the channel realizations. For the resource division approach, the resource sharing between the primary and secondary systems was considered in the time domain.

\section{Results}

Figure 2 presents the performance of the considered techniques in terms of the system sum rate versus FP factor. The system sum rate for all the considered cases were evaluated using eqn. (33) with corresponding capacity equations presented in Section III. The system sum rate capacity in

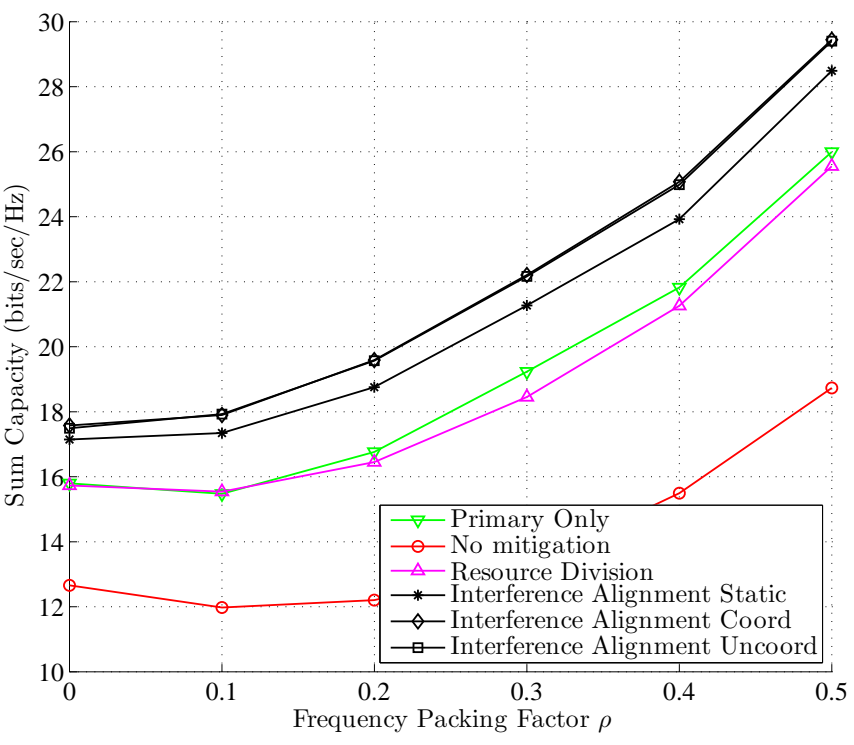

Fig. 2: System sum rate versus frequency packing factor

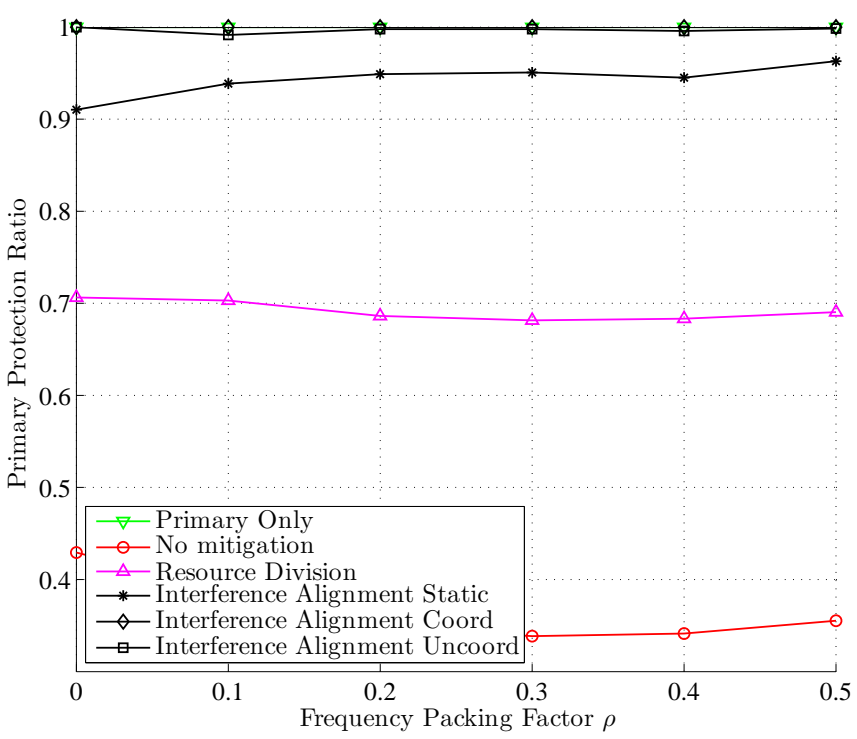

Fig. 3: Primary rate protection ratio versus frequency packing factor

Fig. 2 is the summation of primary capacity and per beam secondary capacity. From the Fig. 2, it can be noted that the IA coordinated and the IA uncoordinated techniques outperform other techniques in terms of the system sum rate. Furthermore, the sum rate increases with the FP factor for all the techniques. It can be concluded that as the frequency packing factor increases, the number of subcarriers which can be placed within the same bandwidth also increases and subsequently, the sum rate is increased.

Figure 3 depicts the $\mathrm{PR}$ versus the FP factor for the considered techniques. The value of $\mathrm{PR}$ for all the considered cases was evaluated using (34) with $C_{\text {po }}$ from (35) and the corresponding $\mathrm{C}_{\mathrm{ps}}$ expressions presented in Section III. It can be noted that the primary rate of the IA coordinated in presence 
of the secondary system perfectly matches the primary only rate and the IA uncoordinated performs slightly worse than the IA coordinated technique. Furthermore, it can be noted that the protection rate for the IA static slightly increases with the value of frequency packing factor and the primary rate is the worst for no-mitigation case. The IA technique achieves improved spectral efciency due to efcient frequency utilization by primary and secondary satellite systems as illustrated in [11]. By examining both the results in Fig. 2 and Fig. 3, it can be concluded that the frequency packing further enhances the spectral efficiency of the IA-based dual satellite coexistence systems on top of the spectral efficiency gain achieved due to IA without causing harmful interference to the primary system.

\section{CONCLUSION}

In this paper, we considered a dual satellite coexistence scenario of multibeam and monobeam satellites with the monobeam satellite as the primary and the multibeam satellite as the secondary. The IA technique has been considered in an underlay cognitive mode in order to mitigate the interference of multibeam satellite terminals towards the monobeam satellite receiver in normal uplink mode. The frequency packing technique has been proposed for the proposed scenario in order to further enhance the spectral efficiency in spectrum-limited satellite applications. The effect of the FP on the performance of the IA coordinated, IA uncoordinated, IA static, resource division and no mitigation techniques has been investigated. With the help of numerical results, it has been shown that the sum rate increases with the value of FP factor for all the considered techniques. Furthermore, it has been noted that the IA coordinated technique achieves the highest sum rate with the perfect primary rate protection and IA uncoordinated technique achieves the highest sum rate with some sacrifice in the primary rate. We consider modeling the combined ACI effect of imperfect bandpass filters and frequency packing phenomenon as our future work.

\section{ACKNOWLEDGEMENT}

This work was supported by the National Research Fund, Luxembourg under the CORE project "CO2SAT: Cooperative and Cognitive Architectures for Satellite Networks".

\section{REFERENCES}

[1] R. Alegre-Godoy, N. Alagha, and M. Vazquez-Castro, "Offered capacity optimization mechanisms for multi-beam satellite systems," in proc. IEEE Int. Conf. Commun., June 2012, pp. 3180 -3184.

[2] S. Chatzinotas, G. Zheng, and B. Ottersten, "Joint precoding with flexible power constraints in multibeam satellite systems," in proc. IEEE GLOBECOM, Dec. 2011, pp. $1-5$.

[3] S. K. Sharma, S. Chatzinotas, and B. Ottersten, "Satellite cognitive communications: Interference modeling and techniques selection," in proc. 6th ASMS/SPSC Conf., Sept. 2012, pp. 111-118.

[4] S. K. Sharma, S. Chatzinotas, and B. Ottersten, "Exploiting polarization for spectrum sensing in cognitive SatComs," in proc. 7th Int. Conf. CROWNCOM, June 2012, pp. 36-41.

[5] S. K. Sharma, S. Chatzinotas, and B. Ottersten, "Spectrum sensing in dual polarized fading channels for cognitive SatComs," in proc. IEEE Globecom Conf., Dec. 2012, pp. 3443-3448.

[6] S. K. Sharma, S. Chatzinotas, and B. Ottersten, "Transmit beamforming for spectral coexistence of satellite and terrestrial networks" to appear in proc. 8th Int. Conf. CROWNCOM, July 2013.
[7] S. K. Sharma, S. Chatzinotas, and B. Ottersten, "Spatial filtering for underlay cognitive SatComs," to appear in proc. 5th Int. Conf. PSATS, June 2013.

[8] S. Kandeepan, L. De Nardis, M.-G. Di Benedetto, A. Guidotti, and G. Corazza, "Cognitive satellite terrestrial radios," in proc. IEEE GLOBECOM, Dec. 2010, pp. $1-6$.

[9] Y. H. Yun and J. H. Cho, "An orthogonal cognitive radio for a satellite communication link," in proc. IEEE 20th Int. Symp. PIMRC, Sept. 2009, pp. $3154-3158$.

[10] L. N. Wang and B. Wang, "Distributed power control for cognitive satellite networks," Advanced Materials Research: Mechatronics and Intelligent Materials II, vol. 71, pp. 1156-1160, March 2012.

[11] S. K. Sharma, S. Chatzinotas, and B. Ottersten, "Interference alignment for spectral coexistence of heterogeneous networks," EURASIP Journal On Wireless Communications and Networking, vol. 2013, no. 46, 2013.

[12] S. K. Sharma, S. Chatzinotas, and B. Ottersten, "Cognitive beamhopping for spectral coexistence of multibeam satellites," to appear in proc. Future Network Mobile Summit 2013, July 2013.

[13] H. Zhou, T. Ratnarajah, and Y.-C. Liang, "On secondary network interference alignment in cognitive radio," in proc. IEEE Symp. New Frontiers in Dynamic Spectrum Access Networks, May 2011, pp. 637 -641 .

[14] S. Kaimaletu, R. Krishnan, S. Kalyani, N. Akhtar, and B. Ramamurthi, "Cognitive interference management in heterogeneous femto-macro cell networks," in proc. IEEE Int. Conf. on Commun., June 2011, pp. $1-6$.

[15] V. Cadambe and S. Jafar, "Interference alignment and the degrees of freedom of wireless X networks," IEEE Trans. Inf. Th., vol. 55, no. 9, pp. $3893-3908$, Sept. 2009.

[16] S. Jafar and S. Shamai, "Degrees of freedom region of the MIMO X channel," IEEE Trans. Inf. Th., vol. 54, no. 1, pp. 151 -170, Jan. 2008.

[17] A. Goldsmith, S. Jafar, I. Maric, and S. Srinivasa, "Breaking spectrum gridlock with cognitive radios: An information theoretic perspective," Proc. IEEE, vol. 97, no. 5, pp. 894 -914, May 2009.

[18] S. Chatzinotas and B. Ottersten, "Cognitive interference alignment between small cells and a macrocell," in proc. 19th Int. Conf. Telecommun., April 2012, pp. 1 -6.

[19] B. Da and R. Zhang, "Exploiting interference alignment in multi-cell cooperative OFDMA resource allocation," in proc. IEEE GLOBECOM 2011, Dec. 2011, pp. $1-5$.

[20] R. Ganesan and A. Klein, "Projection based space-frequency interference alignment in a multi-carrier multi-user two-way relay network," in proc. Int. Symp.on Wireless Commun. Systems, Nov. 2011, pp. 266 -270 .

[21] C. Shi, R. Berry, and M. Honig, "Interference alignment in multi-carrier interference networks," in proc. Int. Symp. Inf. Th., Aug. 2011, pp. 26 -30 .

[22] T. Liu and C. Yang, "Signal alignment for multicarrier code division multiple user two-way relay systems," IEEE Trans. on Wireless Commun., vol. 10 , no. 11 , pp. $3700-3710$, Nov. 2011.

[23] A. Barbieri, D. Fertonani, and G. Colavolpe, "Time-frequency packing for linear modulations: spectral efficiency and practical detection schemes," IEEE Trans. Commun., vol. 57, no. 10, pp. 2951 -2959, Oct. 2009.

[24] J. Zhao and A. Ellis, "Discrete-fourier transform based implementation for optical fast OFDM," in proc. 36th European Conf. and Exhibition on Optical Commun., Sept. 2010, pp. 1 -3.

[25] S. Ibrahim, J. Zhao, D. Rafique, J. O'Dowd, and A. Ellis, "Demonstration of world-first experimental optical fast OFDM system at 7.174 Gbit/s and $14.348 \mathrm{Gbit} / \mathrm{s}$," in proc. 36th European Conf. and Exhibition on Optical Commun., Sept. 2010, pp. 1 -3.

[26] S. Isam and I. Darwazeh, "Characterizing the intercarrier interference of non-orthogonal spectrally efficient FDM system," in proc. 8th Int. Symp. Commun. Systems, Networks Digital Signal Process., July 2012, pp. $1-5$.

[27] M. Hamamura and S. Tachikawa, "Bandwidth efficiency improvement for multi-carrier systems," in proc. 15th IEEE Int. Symp. on Personal, Indoor and Mobile Radio Commun., vol. 1, Sept. 2004, pp. 48 - 52.

[28] H. Yao, T. Royster, J. McLamb, M. Mustafa, and N. Yazdani, "Jitteraware time-frequency resource allocation and packing algorithm," in proc. IEEE Military Commun. Conf., Oct. 2009, pp. 1 -6.

[29] R. Blum, "MIMO capacity with interference," IEEE Journal on Selected Areas in Communications, vol. 21, no. 5, pp. 793 - 801, June 2003. 